\title{
Blowing Up Affine Hybrid Systems
}

\author{
Aaron D. Ames* and Shankar Sastry* \\ Department of EECS \\ University of California at Berkeley \\ Berkeley, CA 94720 \\ \{adames, sastry\}@eecs.berkeley.edu
}

\begin{abstract}
In this paper we construct the "blow up" of an affine hybrid system $\mathbf{H}$, i.e., a new affine hybrid system $\mathrm{Bl}(\mathbf{H})$ in which $\mathrm{H}$ is embedded, that does not exhibit Zeno behavior. We show the existence of a bijection $\Upsilon$ between periodic orbits and equilibrium points of $\mathrm{H}$ and $\mathrm{Bl}(\mathrm{H})$ that preserves stability; we refer to this property as $\mathscr{P}$-stability equivalence.
\end{abstract}

\section{INTRODUCTION}

If $\mathbf{H}$ is an affine hybrid system, we introduce its blow up $\mathrm{Bl}(\mathbf{H})$ which is also an affine hybrid system. The primary benefit of considering $\mathrm{Bl}(\mathbf{H})$ is that it is not Zeno, although its structure suggests many other interesting properties not generally found in affine hybrid systems. In order to demonstrate that $\mathrm{Bl}(\mathbf{H})$ is in some way equivalent to $\mathbf{H}, \mathscr{P}$-stability equivalence is introduced. If $\mathscr{O}^{\mathbf{H}}$ is the set of equilibrium points and periodic orbits of $\mathbf{H}$, then two affine hybrid systems $\mathbf{H}$ and $\mathbf{G}$ are $\mathscr{P}$-stability equivalent if there exists a bijection $\Upsilon: \mathscr{O}^{\mathbf{H}} \rightarrow \mathscr{O}^{\mathbf{G}}$ such that

$$
\mu \in \mathscr{O}^{\mathbf{H}} \text { is } \mathscr{P}_{\text {-stable }} \Leftrightarrow \Upsilon(\mu) \in \mathscr{O}^{\mathbf{G}} \text { is } \mathscr{P} \text {-stable }
$$

where $\mathscr{P}$ is stability in the sense of Lyapunov, asymptotic stability or exponential stability. The purpose of this paper is to prove the following theorem:

Main Theorem: The affine hybrid systems $\mathbf{H}$ and $\mathrm{Bl}(\mathbf{H})$ are $\mathscr{P}$-stability equivalent, and $\mathrm{Bl}(\mathbf{H})$ is not Zeno.

The importance of the Main Theorem is that rather than attempting to determine whether an affine hybrid system is Zeno (which currently is not possible), analysis can be carried out on $\mathrm{Bl}(\mathbf{H})$ where there is no Zeno behavior. Additionally, most analysis on the stability of hybrid systems, or even switched systems, assumes that such systems are not Zeno, cf. [2], [5]-[8]. Because of the Main Theorem, this assumption automatically holds for $\mathrm{Bl}(\mathbf{H})$, and $\mathrm{Bl}(\mathbf{H})$ is $\mathscr{P}$-stability equivalent to $\mathbf{H}$, so the assumption is not restrictive. $\mathrm{Bl}(\mathbf{H})$ displays additional desirable properties that are not found in general affine hybrid systems. Its structure closely resembles a switched system, implying that $\mathrm{Bl}(\mathbf{H})$ might provide a way to apply the analysis carried out on switched systems to affine hybrid systems; since there are considerably more results for switched systems, this would be an important connection. In the future, these and other properties of $\mathrm{Bl}(\mathbf{H})$ will be investigated.

*This research is supported by the National Science Foundation (NSF award number CCR-0225610)

\section{AfFINE HYBRID Systems}

This section introduces the notion of an affine hybrid system. For a more detailed definition, see [1].

2.1 (Discrete states): The set of discrete states is a finite set $Q=\{1, \ldots, m\}$.

2.2 (Domains): The set of domains is the set $D=$ $\left\{D_{i}\right\}_{i \in Q}$, where each $D_{i} \subset \mathbb{R}^{n}$ is an $n$-dimensional affine set, i.e., a set that is affinely constrained. For each set $D_{i}$, there exists a matrix $A_{i} \in \mathbb{R}^{k_{i} \times n}$ and a vector $a_{i} \in \mathbb{R}^{k_{i}}$ such that $x \in D_{i}$ if and only if $A_{i} x+a_{i} \geq 0$, where $k_{i}$ is the number of $n-1$ dimensional affine sets contained in the boundary of $D_{i}$; these are called the faces of $D_{i}$. The faces of $D_{i}$ can be indexed by introducing the indexing set, $F_{i}=\left\{1, \ldots, k_{i}\right\}$, for $i \in Q$. The $j^{t h}$ face of $D_{i}$ is denoted by $\operatorname{Face}_{j}\left(D_{i}\right)$, where $j \in F_{i}$. We can pick an indexing of the faces of $D_{i}$ by letting $\operatorname{Face}_{j}\left(D_{i}\right)$ be the affine set determined by the $j^{\text {th }}$ row of $A_{i}$. More precisely, if $\left(A_{i}\right)_{j *}$ is the $j^{\text {th }}$ row of $A_{i}$ and $\left(a_{i}\right)_{j}$ is the $j^{t h}$ entry of $a_{i}$, then

$$
\begin{aligned}
& x \in \operatorname{Face}_{j}\left(D_{i}\right) \\
& \left(\begin{array}{c}
A_{i} \\
-\left(A_{i}\right)_{j *}
\end{array}\right) x+\left(\begin{array}{c}
a_{i} \\
-\left(a_{i}\right)_{j}
\end{array}\right) \geq 0 .
\end{aligned}
$$

This definition can be extended to affine sets $D_{i}$ with $\operatorname{dim}\left(D_{i}\right) \leq n$ in the obvious manner.

2.3 (Edges): For a set $U$ with $U=\prod_{i=1}^{n} U_{i}$, denote the projections on each of the factors of $U$ by $\pi_{i}: U \rightarrow U_{i}$. Define the set of edges as a set

$$
E \subseteq\{((i, j),(k, l))\}_{(i, j) \in Q \times Q, \quad(k, l) \in F_{i} \times F_{j}},
$$

satisfying the condition that for each $e \in E$, there exists a map $T_{e}(x)=R_{e} x+p_{e}$, with $\left(R_{e}, p_{e}\right) \in S E(n)$, such that $T_{e}\left(\operatorname{Face}_{\pi_{3}(e)}\left(D_{\pi_{1}(e)}\right)\right)=$ Face $_{\pi_{4}(e)}\left(D_{\pi_{2}(e)}\right)$. To simplify notation, write $\operatorname{Source}(e)=\operatorname{Face}_{\pi_{3}(e)}\left(D_{\pi_{1}(e)}\right)$ and Target $(e)=$ Face $_{\pi_{4}(e)}\left(D_{\pi_{2}(e)}\right)$. Given an edge $e \in E$, the affine transformation $T_{e}(x)=R_{e} x+p_{e}$ from $\operatorname{Source}(e)$ to Target $(e)$ is called the transition map. The set of transition maps is the set $T=\left\{T_{e}\right\}_{e \in E}$.

2.4 (Vector fields): A set of vector fields is a set $V=$ $\left\{V_{i}\right\}_{i \in Q}$, where $V_{i}$ is a Lipschitz vector field on $\mathbb{R}^{n}$. The flow of $V_{i}$ on $D_{i}$ is denoted by $\varphi_{i}(t, x)$ for $x \in D_{i}$.

Definition 2.1: An affine hybrid system is a tuple

$$
\mathbf{H}=(Q, D, E, V) .
$$


Note 2.1: From this point on, for the sake of brevity, we will refer to "affine hybrid systems" as "hybrid systems." When dealing with multiple hybrid systems, the superscripts are added to avoid confusion between the hybrid systems. For example, two hybrid systems $\mathbf{H}$ and $\mathbf{G}$ are given by the tuples $\mathbf{H}=\left(Q^{\mathbf{H}}, D^{\mathbf{H}}, E^{\mathbf{H}}, V^{\mathbf{H}}\right)$ and $\mathbf{G}=$ $\left(Q^{\mathbf{G}}, D^{\mathbf{G}}, E^{\mathbf{G}}, V^{\mathbf{G}}\right)$.

2.5: If for some $e \in E, T_{e}(x)=x$, then we say that the transition map associated with the edge $e$ is the identity map. This implies that $\operatorname{Source}(e)=$ Target $(e)$. Since these are affine sets, we can define a matrix $A_{e}$ and vector $a_{e}$ such that $A_{e} x+a_{e} \geq 0$ iff $x \in \operatorname{Source}(e)=\operatorname{Target}(e)$. In particular, these affine constraints could be the affine constraints determining Source $(e)$ or Target $(e)$ as given by Equation (1).

A very special class of hybrid systems is the class hybrid systems in which every transition map is the identity. This is the class of hybrid systems we will consider in this paper; hence we make the following assumption.

Assumption 2.1: For the hybrid system H, every transition map is the identity.

This assumption is not as restrictive as one might think due to the main theorem of [1].

\section{From ExECUTIONS TO $\mathscr{P}$-STABILITY EQUIVALENCE}

This section begins with the definition of a hybrid execution which varies somewhat from the standard definition (cf. [10],[11]). With this definition the hybrid flow can be defined; it is analogous to the flow of a dynamical system. Using this, the important types of equilibrium points and periodic orbits of hybrid systems are introduced, and the different forms of stability that these objects can display are discussed. This section culminates with the definition of $\mathscr{P}$-stability equivalence.

3.1 (Hybrid Execution): Let $\Lambda$ be a finite or countably infinite indexing set such that if $N=|\Lambda|-1$ then $\Lambda=$ $\{0,1, \ldots, N\}$ if $N$ is finite, and $\Lambda=\mathbb{Z}^{*}=\{0,1, \ldots\}$ if $N=\infty$. Also define $\Lambda_{+}=\{1, \ldots, N\}$ if $N$ is finite and $\Lambda_{+}=\mathbb{Z}^{+}=\{1,2, \ldots\}$ if $N=\infty$.

A hybrid time sequence is a finite or infinite sequence of real numbers $\tau=\left\{\tau_{i}\right\}_{i \in \Lambda}$, with $0=\tau_{0} \leq \tau_{1} \leq \cdots \leq \tau_{i} \leq$ $\cdots$, a hybrid edge sequence $\eta=\left\{\eta_{i}\right\}_{i \in \Lambda_{+}}$is a sequence of edges $\eta_{i} \in E$, and a sequence of initial conditions is a sequence $\xi=\left\{\xi_{i}\right\}_{i \in \Lambda}$ with $\xi_{i} \in \mathbb{R}^{n}$.

A hybrid execution is a tuple $\chi=(\tau, \eta, \xi)$ satisfying the following conditions:

For all $0 \leq i<N$,

$\tau_{i+1}=\min \left\{t \geq \tau_{i}: \varphi_{\pi_{1}\left(\eta_{i+1}\right)}\left(t-\tau_{i}, \xi_{i}\right) \in \partial D_{\pi_{1}\left(\eta_{i+1}\right)}\right\}$

$\xi_{i+1}=T_{\eta_{i+1}}\left(\varphi_{\pi_{1}\left(\eta_{i+1}\right)}\left(\tau_{i+1}-\tau_{i}, \xi_{i}\right)\right) \in \operatorname{Target}\left(\eta_{i+1}\right)$,

and $\pi_{1}\left(\eta_{i+1}\right)=\pi_{2}\left(\eta_{i}\right)$ for $1 \leq i<N$.

If $N$ is finite, define an additional element $\tau_{N+1}$ as follows: if $\varphi_{\pi_{2}\left(\eta_{N}\right)}\left(t-\tau_{N}, \xi_{N}\right) \in \partial D_{\pi_{2}\left(\eta_{N}\right)}$ for some finite
$t>\tau_{N}$, then define

$\tau_{N+1}=\min \left\{t>\tau_{N}: \varphi_{\pi_{2}\left(\eta_{N}\right)}\left(t-\tau_{N}, \xi_{N}\right) \in \partial D_{\pi_{2}\left(\eta_{N}\right)}\right\}$,

otherwise set $\tau_{N+1}=\infty$. The element $\tau_{N+1}$ is the termination time of a finite execution.

3.2: Given a hybrid execution $\chi$, the map $d: \Lambda \rightarrow Q$, defined by $d(i)=\pi_{1}\left(\eta_{i+1}\right)$ if $0 \leq i<N$ and $d(N)=$ $\pi_{2}\left(\eta_{N}\right)$ if $N$ is finite, is the discrete state evolution. It sometimes is convenient to associate to an $\eta_{i} \in \eta$ the corresponding edge in $E$, therefore if $E=\left\{e_{1}, \ldots, e_{k}\right\}$, define a map $\rho: \Lambda_{+} \rightarrow\{1, \ldots, k\}$ such that $\eta_{i}=e_{\rho(i)}$; this can be thought of as the evolution of edges.

3.3: An execution $\chi$ is called finite if $N$ is finite and infinite if $N=\infty$. Let

$$
\begin{aligned}
S(\mathbf{H}) & =\{\text { Set of executions of } \mathbf{H}\} \\
S_{0}(\mathbf{H}) & =\{\text { Set of finite executions of } \mathbf{H}\} \\
S_{\infty}(\mathbf{H}) & =\{\text { Set of infinte executions of } \mathbf{H}\} .
\end{aligned}
$$

An execution $\chi$ is Zeno if $\chi \in S_{\infty}(\mathbf{H})$ and $\lim _{i \rightarrow \infty} \tau_{i}=$ $\tau_{\infty}$, where $\tau_{\infty}$ is a finite real number. A hybrid system $\mathbf{H}$ is Zeno if there is at least one Zeno execution. There have been numerous attempts to determine which hybrid systems display Zeno behavior, cf. [4], [6], [11]. When multiple hybrid executions are being discussed (possibly of different hybrid systems), we will write $d^{\chi}, \rho^{\chi}, N^{\chi}, \Lambda^{\chi}$ and $\mathcal{T} \chi$ to remove ambiguity.

3.4: Since we are assuming that $T_{e}=$ id for every $e \in E$, given an execution $\chi=(\tau, \eta, \xi)$ we can define the hybrid flow of $\chi$ which is roughly analogous to the flow of a differential equation. Let

$a_{i}^{\chi}(t)=\left\{\begin{array}{ccc}1 & \text { if } & t \in\left[\tau_{i}, \tau_{i+1}\right], 0 \leq i<N^{\chi} \\ 1 & \text { if } & t \in\left[\tau_{N}, \tau_{N+1}\right), i=N^{\chi}, \quad \chi \in S_{0}(\mathbf{H}) \\ 0 & & \text { otherwise }\end{array}\right.$

and set $\mathcal{T}^{\chi}=\left\{t \in \mathbb{R}: a_{i}^{\chi}(t)>0\right.$, for some $\left.i \in \Lambda^{\chi}\right\}$. Define the hybrid flow as

$$
\varphi^{\chi}\left(t, \xi_{0}\right)=\frac{1}{\sum_{i \in \Lambda} a_{i}^{\chi}(t)} \sum_{i \in \Lambda} a_{i}^{\chi}(t) \varphi_{d \chi(i)}\left(t-\tau_{i}, \xi_{i}\right),
$$

for $t \in \mathcal{T}^{\chi}$. This implies that $\varphi\left(t, \xi_{0}\right)=\varphi_{d \chi(i)}\left(t-\tau_{i}, \xi_{i}\right)$ when $t \in\left[\tau_{i}, \tau_{i+1}\right]$. Note that hybrid flows are defined uniquely by an execution.

3.5: Hybrid systems display more types of equilibrium points and periodic orbits than classical dynamical systems (cf. [8],[9]). We will consider the following:

CSEP $=$ Continuous state equilibrium point: A point $x^{*}$ such that $V_{i}^{\mathbf{H}}\left(x^{*}\right)=0$ for some $i \in Q$.

DSPO $=$ Discrete state periodic orbit: A point $x^{*}$ such that for every execution $\chi \in S_{\infty}(\mathbf{H})$ with $\xi_{0}=x^{*}, \tau_{i}=0$ for all $i \in \Lambda^{\chi}$ and

$$
d(i)=d\left(i+p K^{\chi}\right) \quad d(i) \neq d(j) \quad j \neq i+p K^{\chi}
$$

for some integer $K^{\chi}>0$ (dependent on $\chi$ ) and all $p \in \mathbb{Z}^{*}$. Discrete state periodic orbits can imply the existence of Zeno executions. 
CSPO = Continuous state periodic orbit: A set $\gamma \subset D_{i}^{\mathbf{H}}$ (not a point) that is a periodic orbit of $V_{i}^{\mathbf{H}}$, i.e., there exists a finite $T$ such that for each $\xi_{0} \in \gamma, \varphi_{i}\left(p T, \xi_{0}\right)=\xi_{0}$ for all $p \in \mathbb{Z}$.

MSPO $=$ Mixed state periodic orbit: A connected set $\gamma \subset$ $\mathbb{R}^{n}$ (not a point) such that

$$
\gamma=\bigcup_{\substack{\chi \in S_{\infty}(\mathbf{H}) \\ \xi_{0} \in \gamma}}\left\{\varphi^{\chi}\left(t, \xi_{0}\right): t \in \mathbb{R}^{+}\right\},
$$

where for every $\chi \in S_{\infty}(\mathbf{H})$ with $\xi_{0} \in \gamma, \mathcal{T}^{\chi}=\mathbb{R}^{+}$and

$$
\begin{aligned}
\varphi^{\chi}\left(t, \xi_{0}\right) & =\varphi^{\chi}\left(t+p T^{\chi}\right) & \varphi^{\chi}(t) \in \gamma & \forall t \in \mathbb{R}^{+} \\
d(i) & =d\left(i+p K^{\chi}\right) & d(i) \neq d(j) & j \neq i+p K^{\chi}
\end{aligned}
$$

for a real number $T^{\chi}>0$, an integer $K^{\chi}>0$ (dependent on $\chi$ ) and all $p \in \mathbb{Z}^{*}$.

It is useful to talk about the set of all equilibrium points and periodic orbits of this form. Let

$$
\mathscr{O}^{\mathbf{H}}=\left\{\begin{array}{c}
\text { CSEP's, DSPO's, CSPO's } \\
\text { and MSPO's of } \mathbf{H}
\end{array}\right\} .
$$

If $\mu \in \mathscr{O}^{\mathbf{H}}$ then $\mu$ is either a point (in which case it is a CSEP or a DSPO ), or it is a set not equal to a point (in which case it is a CSPO or a MSPO ).

3.6: Let $B_{\delta}(\mu)$ be a neighborhood of $\mu \in \mathscr{O}^{\mathbf{H}}$, i.e., for all $x \in B_{\delta}(\mu),\|x-\mu\|=\min _{u \in \mu}\|x-u\|<\delta$. Consider the following forms of stability of $\mu$ :

For all $\chi \in S(\mathbf{H})$ with $\xi_{0} \in B_{\delta}(\mu), \mu \in \mathscr{O}^{\mathbf{H}}$ is

$\mathbf{L Y P}=$ Stable in the sense of Lyapunov: If there exists an $\epsilon>0$ such that for all $t \in \mathcal{T} \chi$

$$
\left\|\varphi^{\chi}\left(t, \xi_{0}\right)-\mu\right\| \leq \epsilon .
$$

ASY = Asymptotically stable: If it is LYP and

$$
\lim _{t \rightarrow \sup \mathcal{T} \chi}\left\|\varphi^{\chi}\left(t, \xi_{0}\right)-\mu\right\| \rightarrow 0 .
$$

$\mathbf{E X P}=$ Exponentially stable: If there exists and $\alpha, M>0$ such that for all $t \in \mathcal{T} \chi$

$$
\left\|\varphi^{\chi}\left(t, \xi_{0}\right)-\mu\right\| \leq M e^{-\alpha t}\left\|\xi_{0}-\mu\right\|
$$

A stability property is denoted by $\mathscr{P}=\mathbf{L Y P}, \mathbf{A S Y}$, or EXP.

Definition 3.1: Two hybrid systems $\mathbf{H}$ and $\mathbf{G}$ are $\mathscr{P}$ stability equivalent if there exists a bijection $\Upsilon: \mathscr{O}^{\mathbf{H}} \rightarrow \mathscr{O}^{\mathbf{G}}$ such that $\mu$ is $\mathscr{P}$-stable if and only if $\Upsilon(\mu)$ is $\mathscr{P}$-stable.

\section{The Blow Up of a Hybrid System}

In this section the blow up of a hybrid system is defined constructively. The underlying idea is simple and, as the name suggests, was originally motivated by the blow up of a singular variety in algebraic geometry (more specifically, it was originally motivated by the example on page 28 of Hartshorne's Algebraic Geometry [3]).

4.1 (Construction of $\mathrm{Bl}(\mathbf{H})$ ): The blow up of a hybrid system $\mathbf{H}$ is a hybrid system

$$
\mathrm{Bl}(\mathbf{H})=\left(Q^{\mathrm{Bl}(\mathbf{H})}, D^{\mathrm{Bl}(\mathbf{H})}, E^{\mathrm{Bl}(\mathbf{H})}, V^{\mathrm{Bl}(\mathbf{H})}\right),
$$

where the individual elements are defined as follows: $Q^{\mathrm{Bl}(\mathbf{H})}$ : If $Q^{\mathbf{H}}=\{1, \ldots, m\}$ and $k=\left|E^{\mathbf{H}}\right|$, then

$$
Q^{\mathrm{Bl}(\mathbf{H})}=\{1, \ldots, m+k\} .
$$

$\underline{D^{\mathrm{Bl}(\mathbf{H})}}$ : Let $q \in \mathbb{R}^{m}$ and $\lambda_{i}$ be the $i^{t h}$ standard basis vector of $\mathbb{R}^{m}$. If $i \leq m$, then $D_{i}^{\mathbf{H}}$ is determined by the affine constraints $A_{i}^{\mathbf{H}} x+a_{i}^{\mathbf{H}}$. Define $D_{i}^{\mathrm{Bl}(\mathbf{H})}$ to be the affine set given by the affine constraints

$$
\left(\begin{array}{cc}
A_{i}^{\mathbf{H}} & 0 \\
0 & I \\
0 & -I
\end{array}\right)\left(\begin{array}{c}
x \\
q
\end{array}\right)+\left(\begin{array}{c}
a_{i}^{\mathbf{H}} \\
-\lambda_{i} \\
\lambda_{i}
\end{array}\right) \geq 0 .
$$

By indexing the elements of $E^{\mathbf{H}}$ such that $\left\{e_{1}^{\mathbf{H}}, \ldots, e_{k}^{\mathbf{H}}\right\}=$ $E^{\mathbf{H}}$, for $i \in\{m+1, \ldots, m+k\}$, we can define $D_{i}^{\mathrm{Bl}(\mathbf{H})}$ to be the affine set given by the affine constraints

$$
\begin{aligned}
& \left(\begin{array}{cc}
0 & -I \\
0 & \lambda_{\pi_{1}\left(e_{i-m}^{\mathbf{H}}\right)}^{T}+\lambda_{\pi_{2}\left(e_{i-m}^{\mathbf{H}}\right)}^{T} \\
0 & -\lambda_{\pi_{1}\left(e_{i-m}^{\mathbf{H}}\right)}^{T}-\lambda_{\pi_{2}\left(e_{i-m}^{\mathbf{H}}\right)}^{T} \\
0 & \multicolumn{1}{c}{{ }^{T}}
\end{array}\right)\left(\begin{array}{c}
x \\
q
\end{array}\right) \\
& +\left(\begin{array}{c}
\lambda_{\pi_{1}\left(e_{i-m}^{\mathbf{H}}\right)}+\lambda_{\pi_{2}\left(e_{i-m}^{\mathbf{H}}\right)} \\
-1 \\
1 \\
0 \\
a_{e_{i-m}^{\mathbf{H}}}^{\mathbf{H}}
\end{array}\right) \geq 0 . \\
& \underline{E^{\mathrm{Bl}(\mathbf{H})}} \text { : Again letting }\left\{e_{1}^{\mathbf{H}}, \ldots, e_{k}^{\mathbf{H}}\right\}=E^{\mathbf{H}} \text {, define } \\
& E^{\mathrm{Bl}(\mathbf{H})}=\left\{e_{1}^{\mathrm{Bl}(\mathbf{H})}, \ldots, e_{2 k}^{\mathrm{Bl}(\mathbf{H})}\right\},
\end{aligned}
$$

where

$$
\begin{aligned}
e_{i}^{\mathrm{Bl}(\mathbf{H})} & =\left(\left(\pi_{1}\left(e_{i}^{\mathbf{H}}\right), i+m\right),\left(\pi_{3}\left(e_{i}^{\mathbf{H}}\right), \pi_{1}\left(e_{i}^{\mathbf{H}}\right)\right),\right. \\
e_{i+k}^{\mathrm{Bl}(\mathbf{H})} & =\left(\left(i+m, \pi_{2}\left(e_{i}^{\mathbf{H}}\right)\right),\left(\pi_{2}\left(e_{i}^{\mathbf{H}}\right), \pi_{4}\left(e_{i}^{\mathbf{H}}\right)\right),\right.
\end{aligned}
$$

for $1 \leq i \leq k$. By construction it follows that $T_{e_{1}^{\mathrm{Bl}(\mathbf{H})}}=$ $\cdots=T_{e_{2 k}^{\mathrm{Bl}(\mathbf{H})}}=\mathrm{id}$.

$\underline{V^{\mathrm{Bl}(\mathbf{H})}}$ : Write $\tilde{x}=(x, q) \in \mathbb{R}^{n+m}$, and define

$$
V_{i}^{\mathrm{Bl}(\mathbf{H})}(\tilde{x})=\left(\begin{array}{c}
V_{i}^{\mathbf{H}}(x) \\
0
\end{array}\right)
$$

for $1 \leq i \leq m$, and

$$
V_{i}^{\mathrm{Bl}(\mathbf{H})}(\tilde{x})=\left(\begin{array}{c}
0 \\
\lambda_{\pi_{2}\left(e_{i-m}^{\mathbf{H}}\right)}-\lambda_{\pi_{1}\left(e_{i-m}^{\mathbf{H}}\right)}
\end{array}\right)
$$

for $m+1 \leq i \leq m+k$. To avoid confusion, let $\psi_{i}(t, \tilde{x})$ be the solution to $V_{i}^{\mathrm{Bl}(\mathbf{H})}$ for $\tilde{x} \in D_{i}^{\mathrm{Bl}(\mathbf{H})}$.

\section{Relationships Between $\mathbf{H}$ and $\mathrm{Bl}(\mathbf{H})$}

In this section, several relationships between $\mathbf{H}$ and $\mathrm{Bl}(\mathbf{H})$ are established. These are important in that they show that in some sense the qualitative behavior of $\mathbf{H}$ and $\mathrm{Bl}(\mathbf{H})$ are the same. More specifically, it is shown that there is an injective map from $S(\mathbf{H})$ to $S(\mathrm{Bl}(\mathbf{H}))$ and it is given 
explicitly. This is used to establish a bijection between $\mathscr{O}^{\mathbf{H}}$ and $\mathscr{O}^{\mathrm{Bl}(\mathbf{H})}$ which is again given explicitly.

5.1: To determine a map between $D_{i}^{\mathbf{H}}$ and $D_{i}^{\mathrm{Bl}(\mathbf{H})}$ consider the maps

$$
D_{i}^{\mathbf{H}} \stackrel{\iota_{i}}{\longrightarrow} \coprod_{i \in Q^{\mathbf{H}}} D_{i}^{\mathbf{H}} \stackrel{\Gamma}{\longrightarrow} \bigcup_{i \in Q^{\mathbf{H}}} D_{i}^{\mathrm{Bl}(\mathbf{H})}
$$

where $\iota_{i}(x)=(x, i)$ and $\Gamma((x, i))=\left(x, \lambda_{i}\right)^{T} \in \mathbb{R}^{n+m}$ Define $\Gamma_{i}=\Gamma \circ \iota_{i}$; this is the desired map from $D_{i}^{\mathbf{H}}$ to $D_{i}^{\mathrm{Bl}(\mathbf{H})}$. Note that $D_{i}^{\mathrm{Bl}(\mathbf{H})}=\Gamma_{i}\left(D_{i}^{\mathbf{H}}\right)$ and $\Gamma_{i}$ has a left inverse given by $\pi_{x}\left(x, \lambda_{i}\right)=x$.

Proposition 5.1: There exists an injective map

$$
\Xi: S(\mathbf{H}) \longrightarrow S(\mathrm{Bl}(\mathbf{H}))
$$

with a closed form solution.

Proof: Let $\chi=(\tau, \eta, \xi) \in S(\mathbf{H})$ and denote the image of $\Xi$ by $\Xi(\chi)=\left(\Xi_{1}(\tau), \Xi_{2}(\eta), \Xi_{3}(\xi)\right)$. Let $d^{\chi}$ and $\rho^{\chi}$ be the discrete state evolution and the evolution of edges for the execution $\chi$ : see Paragraph 3.2. Now let

$$
\Lambda^{\Xi(\chi)}=\left\{\begin{array}{ccc}
\left\{0,1, \ldots, 2 N^{\chi}\right\} & \text { if } & \chi \in S_{0}(\mathbf{H}) \\
\Lambda^{\chi} & \text { otherwise }
\end{array}\right.
$$

and define the map $\Xi$ as:

$$
\begin{aligned}
& \Xi_{1}(\tau)_{i}=\left\{\begin{array}{cl}
\tau_{\frac{i}{2}}+\frac{i}{2} & \text { if } \mathrm{i} \text { even } \\
\tau_{\frac{i+1}{2}}+\frac{i-1}{2} & \text { if } \mathrm{i} \text { odd }
\end{array}\right. \\
& \Xi_{2}(\eta)_{i}=\left\{\begin{array}{ccc}
e_{\rho \chi\left(\frac{i}{2}\right)+k}^{\mathrm{Bl}(\mathbf{H})} & \text { if } \mathrm{i} \text { even } \\
e_{\rho \chi\left(\frac{i+1}{2}\right)}^{\mathrm{Bl}(\mathbf{H})} & \text { if } \mathrm{i} \text { odd }
\end{array}\right. \\
& \Xi_{3}(\xi)_{i}=\left\{\begin{array}{cl}
\Gamma_{d \chi\left(\frac{i}{2}\right)}\left(\xi_{\frac{i}{2}}\right) & \text { if } \mathrm{i} \text { even } \\
\Gamma_{d \chi\left(\frac{i-1}{2}\right)}\left(\xi_{\left.\frac{i+1}{2}\right)}\right) & \text { if } \mathrm{i} \text { odd }
\end{array}\right.
\end{aligned}
$$

The associated discrete state evolution and the evolution of edges for $\Xi$ are given by

$$
\begin{aligned}
& d^{\Xi(\chi)}(i)=\left\{\begin{array}{cc}
d^{\chi}\left(\frac{i}{2}\right) & \text { if } \mathrm{i} \text { even } \\
\rho^{\chi\left(\frac{i-1}{2}\right)}+m & \text { if } \mathrm{i} \text { odd }
\end{array}\right. \\
& \rho^{\Xi(\chi)}(i)=\left\{\begin{array}{cc}
\rho^{\chi}\left(\frac{i}{2}\right)+k & \text { if } \mathrm{i} \text { even } \\
\rho^{\chi\left(\frac{i+1}{2}\right)} & \text { if } \text { i odd }
\end{array}\right.
\end{aligned}
$$

It can be verified that $\Xi$ is injective as desired.

Corollary 5.1: There is a bijection of sets

$$
\begin{gathered}
\overline{S_{\infty}(\mathrm{Bl}(\mathbf{H}))} \\
\left.S_{\infty}(\mathbf{H}) \longleftrightarrow \quad \begin{array}{c}
\tilde{\chi} \in S_{\infty}(\mathrm{Bl}(\mathbf{H})) \text { with } \\
\tilde{\xi}_{0} \in D_{i}^{\mathrm{Bl}(\mathbf{H})} \text { for } i \in Q^{\mathbf{H}}
\end{array}\right\}
\end{gathered}
$$

5.2: The case given in Corollary 5.1 will be of the most interest. For $\tilde{\chi} \in \overline{S_{\infty}(\mathrm{Bl}(\mathbf{H}))}$ and for $\chi$ such that $\tilde{\chi}=\Xi(\chi)$, if $t \in\left[\tilde{\tau}_{2 i}, \tilde{\tau}_{2 i+1}\right]$ we can write $\psi^{\tilde{\chi}}$ as

$$
\begin{aligned}
\psi^{\tilde{\chi}}\left(t, \tilde{\xi}_{0}\right) & =\psi_{d \tilde{\chi}(2 i)}\left(t-\tilde{\tau}_{2 i}, \tilde{\xi}_{2 i}\right) \\
& =\Gamma_{d^{\chi}(i)}\left(\varphi_{d \chi(i)}\left(t-\tilde{\tau}_{2 i}, \xi_{i}\right)\right),
\end{aligned}
$$

and for $t \in\left[\tilde{\tau}_{2 i-1}, \tilde{\tau}_{2 i}\right], \psi^{\tilde{\chi}}$ is given by

$$
\begin{aligned}
& \psi^{\tilde{\chi}}\left(t, \tilde{\xi}_{0}\right)=\psi_{d \tilde{\chi}(2 i-1)}\left(t-\tilde{\tau}_{2 i-1}, \tilde{\xi}_{2 i-1}\right) \\
& \quad=\left(1-t+\tilde{\tau}_{2 i-1}\right) \Gamma_{d \times(i-1)}\left(\xi_{i}\right)+\left(t-\tilde{\tau}_{2 i}\right) \Gamma_{d \times(i)}\left(\xi_{i}\right) .
\end{aligned}
$$

Proposition 5.2: There are the following bijections:

$$
\{\text { CSEP's of } \mathbf{H}\} \longleftrightarrow \quad\{\text { CSEP's of } \mathrm{Bl}(\mathbf{H})\}
$$

$\{$ CSPO's of $\mathbf{H}\} \quad \longleftrightarrow \quad$ CSPO's of $\mathrm{Bl}(\mathbf{H})\}$

$\{$ DSPO's of $\mathbf{H}\} \longleftrightarrow\left\{\begin{array}{c}\text { MSPO's of } \mathrm{Bl}(\mathbf{H}) \text { with } \\ \tilde{\gamma} \subseteq \bigcup_{i \in Q^{\mathrm{Bl}(\mathbf{H})} \backslash Q^{\mathbf{H}}} D_{i}^{\mathrm{Bl}(\mathbf{H})}\end{array}\right\}$

$\{$ MSPO's of $\mathbf{H}\} \longleftrightarrow\left\{\begin{array}{c}\text { MSPO's of } \mathrm{Bl}(\mathbf{H}) \text { with } \\ \tilde{\gamma} \nsubseteq \bigcup_{i \in Q^{\mathrm{Bl}(\mathbf{H})} \backslash Q^{\mathbf{H}}} D_{i}^{\mathrm{Bl}(\mathbf{H})}\end{array}\right\}$

Proof: The first and second bijections are clear. First let us verify the fourth bijection.

Now if $\gamma$ is a MSPO of $\mathbf{H}$, the claim is that

$$
\begin{aligned}
\Upsilon(\gamma)= & \bigcup_{\substack{\chi \in S_{\infty}(\mathbf{H}) \\
\text { with } \xi_{0} \in \gamma}}\left(\bigcup_{i=1}^{K^{\chi}} \operatorname{ccl}\left\{\Gamma_{d \chi(i-1)}\left(\xi_{i}\right), \Gamma_{d \chi(i)}\left(\xi_{i}\right)\right\}\right. \\
& \left.\cup \bigcup_{i=0}^{K^{\chi}}\left\{\Gamma_{d \chi(i)}\left(\varphi_{d \chi(i)}\left(t-\tau_{i}, \xi_{i}\right)\right)\right\}_{t \in\left[\tau_{i}, \tau_{i+1}\right]}\right)
\end{aligned}
$$

is a bijection, where "ccl" is the convex closure. Setting $\Upsilon^{-1}=\pi_{x}$, clearly $\Upsilon^{-1} \circ \Upsilon=\mathrm{id}$.

To verify that $\Upsilon \circ \Upsilon^{-1}=\mathrm{id}$, let $\tilde{\gamma}$ be a MSPO of $\operatorname{Bl}(\mathbf{H})$ with $\tilde{\gamma} \nsubseteq \bigcup_{i \in Q^{\mathrm{Bl}(\mathbf{H})} \backslash Q^{\mathbf{H}}} D_{i}^{\mathrm{Bl}(\mathbf{H})}$. Consider an execution $\tilde{\chi} \in$ $\overline{S_{\infty}(\mathrm{Bl}(\mathbf{H}))}$ with $\psi \tilde{\chi}\left(t, \tilde{\xi}_{0}\right) \subseteq \tilde{\gamma}$. By Corollary 5.1 there exists a $\chi$ with $\tilde{\chi}=\Xi(\chi)$ and $K^{\tilde{\chi}}=2 K^{\chi}$. Referring to Paragraph 5.2 there are the following relations

$$
\begin{aligned}
& \left\{\psi^{\tilde{\chi}}\left(t, \tilde{\xi}_{0}\right)\right\}_{t \in \mathbb{R}^{+}}=\bigcup_{i=0}^{K^{\tilde{\chi}}}\left\{\psi_{d \tilde{\chi}(i)}\left(t-\tilde{\tau}_{i}, \tilde{\xi}_{i}\right)\right\}_{t \in\left[\tilde{\tau}_{i}, \tilde{\tau}_{i+1}\right]} \\
& =\bigcup_{i=1}^{K^{\chi}}\left\{\psi_{d \tilde{\chi}(2 i-1)}\left(t-\tilde{\tau}_{2 i-1}, \tilde{\xi}_{2 i-1}\right)\right\}_{t \in\left[\tilde{\tau}_{2 i-1}, \tilde{\tau}_{2 i}\right]} \\
& \cup \bigcup_{i=0}^{K^{\chi}}\left\{\psi_{d \tilde{\chi}(2 i)}\left(t-\tilde{\tau}_{2 i}, \tilde{\xi}_{2 i}\right)\right\}_{t \in\left[\tilde{\tau}_{2 i}, \tilde{\tau}_{2 i+1}\right]} \\
& =\bigcup_{i=1}^{K^{\chi}}\left\{\left(1-t+\tilde{\tau}_{2 i-1}\right) \Gamma_{d \chi(i-1)}\left(\xi_{i}\right)\right. \\
& \left.+\left(t-\tilde{\tau}_{2 i}\right) \Gamma_{d \times(i)}\left(\xi_{i}\right)\right\}_{t \in\left[\tilde{\tau}_{2 i-1}, \tilde{\tau}_{2 i}\right]} \\
& \cup \bigcup_{i=0}^{K^{\chi}}\left\{\Gamma_{d \chi(i)}\left(\varphi_{d \times(i)}\left(t-\tilde{\tau}_{2 i}, \xi_{i}\right)\right)\right\}_{t \in\left[\tilde{\tau}_{2 i}, \tilde{\tau}_{2 i+1}\right]} \\
& =\bigcup_{i=1}^{K^{\chi}} \operatorname{ccl}\left\{\Gamma_{d \chi(i-1)}\left(\xi_{i}\right), \Gamma_{d^{\chi}(i)}\left(\xi_{i}\right)\right\} \\
& \cup \bigcup_{i=0}^{K^{\chi}}\left\{\Gamma_{d \chi(i)}\left(\varphi_{d \chi(i)}\left(t-\tau_{i}, \xi_{i}\right)\right)\right\}_{t \in\left[\tau_{i}, \tau_{i+1}\right]} .
\end{aligned}
$$


Now $\pi_{x}(\tilde{\gamma})$ is a MSPO of $\mathbf{H}$, and we have

$$
\begin{aligned}
& \tilde{\gamma}=\bigcup_{\substack{\tilde{\chi}_{\infty}(\mathrm{Bl}(\mathbf{H})) \\
\text { with } \tilde{\xi}_{0} \in \tilde{\gamma}}}\left\{\psi^{\tilde{\chi}}\left(t, \tilde{\xi}_{0}\right)\right\}_{t \in \mathbb{R}^{+}}
\end{aligned}
$$

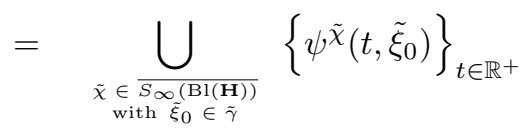

$$
\begin{aligned}
& =\bigcup_{\substack{\chi \in S_{\infty}(\mathbf{H}) \\
\xi_{0} \in \pi_{x}(\tilde{\gamma})}}\left(\bigcup_{i=1}^{K^{\chi}} \operatorname{ccl}\left\{\Gamma_{d^{\chi}(i-1)}\left(\xi_{i}\right), \Gamma_{d^{\chi}(i)}\left(\xi_{i}\right)\right\}\right. \\
& \left.\cup \bigcup_{i=0}^{K^{\chi}}\left\{\Gamma_{d \chi(i)}\left(\varphi_{d \chi(i)}\left(t-\tau_{i}, \xi_{i}\right)\right)\right\}_{t \in\left[\tau_{i}, \tau_{i+1}\right]}\right) \\
& =\Upsilon \circ \Upsilon^{-1}(\tilde{\gamma}) .
\end{aligned}
$$

which proves the fourth bijection.

To prove the third bijection, let $x^{*}$ be a DSPO . The claim is that

$$
\Upsilon\left(x^{*}\right)=\bigcup_{\substack{\chi \in S_{\infty}(\mathbf{H}) \\ \text { with } \\ \xi_{0}=x^{*}}} \bigcup_{i=1}^{K^{\chi}} \operatorname{ccl}\left\{\Gamma_{d \chi(i-1)}\left(x^{*}\right), \Gamma_{d \chi(i)}\left(x^{*}\right)\right\}
$$

is bijective. It will be seen that this is a special case of the fourth bijection.

Let $\tilde{\gamma}$ be a MSPO of $\operatorname{Bl}(\mathbf{H})$ with $\tilde{\gamma} \subseteq$ $\bigcup_{i \in Q^{\mathrm{Bl}(\mathbf{H})} \backslash Q^{\mathbf{H}}} D_{i}^{\mathrm{Bl}(\mathbf{H})}$. Again consider an execution $\tilde{\chi} \in \overline{S_{\infty}(\mathrm{Bl}(\mathbf{H}))}$ with $\psi^{\tilde{\chi}}\left(t, \tilde{\xi}_{0}\right) \subseteq \tilde{\gamma}$; in this case $\tilde{\xi}_{0} \in \partial D_{i}^{\mathrm{Bl}(\mathbf{H})}$ and $\tilde{\xi}_{0}=\Gamma_{d \tilde{\chi}(0)}\left(x^{*}\right)$ for $x^{*}=\pi_{x}(\tilde{\gamma})$ (which is a single point because $\tilde{\gamma}$ is connected). By referring to the construction of $\mathrm{Bl}(\mathbf{H})$ and Paragraph 5.2

$$
\psi_{d \tilde{\chi}(2 i)}\left(t-\tilde{\tau}_{2 i}, \tilde{\xi}_{2 i}\right) \notin \bigcup_{i \in Q^{\mathrm{Bl}(\mathbf{H})} \backslash Q^{\mathbf{H}}} D_{i}^{\mathrm{Bl}(\mathbf{H})},
$$

if $t \neq \tilde{\tau}_{2 i}, \tilde{\tau}_{2 i+1}$. Therefore, $\tilde{\tau}_{2 i}=\tilde{\tau}_{2 i+1}$. By Corollary 5.1, $\tilde{\chi}=\Xi(\chi)$ and for this $\chi, \tilde{\tau}_{2 i}=\tau_{i}=\tau_{i+1}=\tilde{\tau}_{2 i+1}$. This gives

$$
\begin{aligned}
& \left\{\Gamma_{d \chi(i)}\left(\varphi_{d \chi(i)}\left(t-\tau_{i}, \xi_{i}\right)\right)\right\}_{t \in\left[\tau_{i}, \tau_{i+1}\right]} \\
& =\left\{\Gamma_{d \chi(i)}\left(\xi_{i}\right)\right\} \in \operatorname{ccl}\left\{\Gamma_{d \chi(i-1)}\left(\xi_{i}\right), \Gamma_{d \chi(i)}\left(\xi_{i}\right)\right\} .
\end{aligned}
$$

Now since $\pi_{x}(\tilde{\gamma})=x^{*}$ (again because $\tilde{\gamma}$ is connected) and $\tilde{\xi}_{i} \in \tilde{\gamma}, \xi_{i}=\pi_{x}\left(\tilde{\xi}_{2 i}\right)=x^{*}$. Therefore,

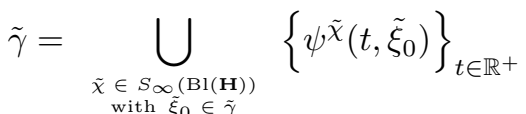

$$
\begin{aligned}
& =\bigcup_{\substack{\chi \in S_{\infty}(\mathbf{H}) \\
\xi_{0}=x^{*}}}\left(\bigcup_{i=1}^{K^{\chi}} \operatorname{ccl}\left\{\Gamma_{d \chi(i-1)}\left(\xi_{i}\right), \Gamma_{d \chi(i)}\left(\xi_{i}\right)\right\}\right. \\
& \left.\cup \bigcup_{i=0}^{K^{\chi}}\left\{\Gamma_{d \chi(i)}\left(\varphi_{d \chi(i)}\left(t-\tau_{i}, \xi_{i}\right)\right)\right\}_{t \in\left[\tau_{i}, \tau_{i+1}\right]}\right) \\
& =\bigcup_{\substack{\chi \in S_{\infty}(\mathbf{H}) \\
\xi_{0}=x^{*}}} \bigcup_{i=1}^{K^{\chi}} \operatorname{ccl}\left\{\Gamma_{d \chi}{ }_{(i-1)}\left(x^{*}\right), \Gamma_{d \chi}{ }_{(i)}\left(x^{*}\right)\right\} \\
& =\Upsilon \circ \Upsilon^{-1}(\tilde{\gamma}) \text {. }
\end{aligned}
$$

This completes the proof.

Proposition 5.3: There is a bijection

$$
\Upsilon: \mathscr{O}^{\mathbf{H}} \longrightarrow \mathscr{O}^{\mathrm{Bl}(\mathbf{H})} \text {. }
$$

Proof: This follows from Proposition 5.2 and from the fact that $\mathrm{Bl}(\mathbf{H})$ has no DSPO's .

\section{IMPORTANT PRoperties OF $\mathrm{Bl}(\mathbf{H})$}

In this section we prove the main results of this paper.

Theorem 1: $\mathrm{Bl}(\mathbf{H})$ has no Zeno executions.

Proof: Suppose that $\mathrm{Bl}(\mathbf{H})$ had a Zeno execution $\tilde{\chi} \in$ $S_{\infty}(\mathrm{Bl}(\mathbf{H}))$; without loss of generality let $\tilde{\chi} \in \overline{S_{\infty}(\mathrm{Bl}(\mathbf{H}))}$. Then $\tilde{\chi}=\Xi(\chi)$ for some execution $\chi$ of $\mathbf{H}$. We are assuming that $\tilde{\chi}$ is Zeno, so $\Xi(\tau)_{i} \leq B$ for some integer $B$ and all $i \in \Lambda^{\Xi(\chi)}$. But

$$
\Xi(\tau)_{2 B+2}=\tau_{\frac{2 B+2}{2}}+\frac{2 B+2}{2} \geq B+1>B
$$

which gives a contradiction.

Theorem 2: $\mathbf{H}$ and $\mathrm{Bl}(\mathbf{H})$ are $\mathscr{P}$-stability equivalent.

Proof: First consider the case when $\mu$ is a CSEP or CSPO. By Proposition 5.2, $\Upsilon(\mu)$ is also a CSEP or a CSPO. If $\mu \subset D_{i}^{\mathbf{H}}$ for $i \in Q^{\mathbf{H}}$, then $\Upsilon(\mu) \subset D_{i}^{\mathrm{Bl}(\mathbf{H})}$ (note that this does not include the degenerate case where $\mu=D_{i}^{\mathbf{H}}$, but the proof of this case is clear). Now pick $\delta$ such that $B_{\delta}(\mu) \subset D_{i}^{\mathbf{H}}$ and $B_{\delta}(\Upsilon(\mu)) \subset D_{i}^{\mathrm{Bl}(\mathbf{H})}$. There is an obvious bijection

$$
\left\{\begin{array}{c}
\chi \in S(\mathbf{H}) \text { with } \\
\xi_{0} \in B_{\delta}(\mu)
\end{array}\right\} \longleftrightarrow\left\{\begin{array}{c}
\chi \in S(\mathrm{Bl}(\mathbf{H})) \text { with } \\
\tilde{\xi}_{0} \in B_{\delta}(\Upsilon(\mu))
\end{array}\right\}
$$

Combining this bijection with the formula for $\Upsilon(\mu)$ given in the proof of Proposition 5.2,

$$
\left\|\psi^{\tilde{\chi}}\left(t, \tilde{\xi}_{0}\right)-\Upsilon(\mu)\right\|=\left\|\varphi^{\chi}\left(t, \xi_{0}\right)-\mu\right\|,
$$

which implies $\mathscr{P}$-stability equivalence.

Now consider the case where $\mu$ is a DSPO or a MSPO; in this case $\Upsilon(\mu)$ is given in the proof of Proposition 5.2. Without loss of generality, only the executions in $S_{\infty}(\mathbf{H})$ and $\overline{S_{\infty}(\mathrm{Bl}(\mathbf{H}))}$ need be considered. For every $\tilde{\chi} \in \overline{S_{\infty}(\mathrm{Bl}(\mathbf{H}))}, \tilde{\chi}=\Xi(\chi)$. By Paragraph 5.2, for $t \in$ $\left[\tilde{\tau}_{2 i}, \tilde{\tau}_{2 i+1}\right]$,

$$
\begin{aligned}
\| \psi^{\tilde{\chi}}\left(t, \tilde{\xi}_{0}\right) & -\Upsilon(\mu) \| \\
& =\left\|\psi_{d \tilde{\chi}(2 i)}\left(t-\tilde{\tau}_{2 i}, \tilde{\xi}_{2 i}\right)-\Upsilon(\mu)\right\| \\
& =\left\|\Gamma_{d \chi(i)}\left(\varphi_{d \chi(i)}\left(t-\tilde{\tau}_{2 i}, \xi_{i}\right)\right)-\Upsilon(\mu)\right\| \\
& =\left\|\varphi_{d \chi(i)}\left(t-\tilde{\tau}_{2 i}, \xi_{i}\right)-\mu\right\| \\
& =\left\|\varphi_{d \chi(i)}\left(t-\tau_{i}-i, \xi_{i}\right)-\mu\right\|
\end{aligned}
$$

and for $t \in\left[\tilde{\tau}_{2 i-1}, \tilde{\tau}_{2 i}\right]$,

$$
\begin{aligned}
\| \psi^{\tilde{\chi}}\left(t, \tilde{\xi}_{0}\right)- & \Upsilon(\mu) \| \\
= & \left\|\psi_{d \tilde{\chi}(2 i-1)}\left(t-\tilde{\tau}_{2 i-1}, \tilde{\xi}_{2 i-1}\right)-\Upsilon(\mu)\right\| \\
= & \|\left(1-t+\tilde{\tau}_{2 i-1}\right) \Gamma_{d \chi(i-1)}\left(\xi_{i}\right) \\
& \quad+\left(t-\tilde{\tau}_{2 i}\right) \Gamma_{d \chi(i)}\left(\xi_{i}\right)-\Upsilon(\mu) \| \\
= & \left\|\xi_{i}-\mu\right\| .
\end{aligned}
$$


Conversely, for $\chi \in S_{\infty}(\mathbf{H})$, and $\tilde{\chi} \in \overline{S_{\infty}(\mathrm{Bl}(\mathbf{H}))}$ such that $\chi=\Omega(\tilde{\chi})$, for $t \in\left[\tau_{i}, \tau_{i+1}\right]$,

$$
\begin{aligned}
\| \varphi^{\chi}\left(t, \xi_{i}\right) & -\mu \| \\
& =\left\|\varphi_{d \chi(i)}\left(t-\tau_{i}, \xi_{i}\right)-\mu\right\| \\
& =\left\|\psi_{d \tilde{\chi}(2 i)}\left(t-\tau_{i}+i, \tilde{\xi}_{2 i}\right)-\Upsilon(\mu)\right\| .
\end{aligned}
$$

To show $\mathscr{P}$-stability equivalence, it must be shown that

$$
\mu \text { is } \mathscr{P}_{\text {-stable }} \Leftrightarrow \Upsilon(\mu) \text { is } \mathscr{P}_{\text {-stable }}
$$

for $\mathscr{P}=$ LYP, ASY, or EXP. Throughout the rest of the proof, let $B_{\delta}(\mu)$ and $B_{\delta}(\Upsilon(\mu))$ be sufficiently small neighborhoods such that $\pi_{x}\left(B_{\delta}(\Upsilon(\mu))\right)=B_{\delta}(\mu)$, and consider only $\chi \in S_{\infty}(\mathbf{H})$ with $\xi_{0} \in B_{\delta}(\mu)$ and $\tilde{\chi} \in$ $\overline{S_{\infty}(\mathrm{Bl}(\mathbf{H}))}$ with $\tilde{\xi}_{0} \in B_{\delta}(\Upsilon(\mu))$.

$\mathscr{P}=$ LYP: $(\Leftrightarrow)$ Follows from (2), (3) and (4).

$\mathscr{P}=\mathbf{A S Y}$ : We just showed that $\mu$ is LYP if and only if $\Upsilon(\mu)$ is LYP, so it only remains to show that

$$
\begin{gathered}
\lim _{i \rightarrow \infty}\left\|\varphi^{\chi}\left(\tau_{i}, \xi_{0}\right)-\mu\right\| \rightarrow 0 \\
\lim _{i \rightarrow \infty}\left\|\tilde{\psi}^{\mathbb{\chi}}\left(\tilde{\tau}_{i}, \tilde{\xi}_{0}\right)-\Upsilon(\mu)\right\| \rightarrow 0
\end{gathered}
$$

$(\Rightarrow)$ Because

$$
\left\|\tilde{\xi}_{2 i-1}-\Upsilon(\mu)\right\|=\left\|\tilde{\xi}_{2 i}-\Upsilon(\mu)\right\|=\left\|\xi_{i}-\mu\right\|,
$$

it follows that

$$
\begin{aligned}
\lim _{i \rightarrow \infty}\left\|\psi^{\tilde{\chi}}\left(\tilde{\tau}_{2 i}, \xi_{0}\right)-\Upsilon(\mu)\right\| \\
=\lim _{i \rightarrow \infty}\left\|\psi^{\tilde{\chi}}\left(\tilde{\tau}_{2 i-1}, \xi_{0}\right)-\Upsilon(\mu)\right\| \\
=\lim _{i \rightarrow \infty}\left\|\xi_{i}-\mu\right\| \\
=\lim _{i \rightarrow \infty}\left\|\varphi^{\chi}\left(\tau_{i}, \xi_{0}\right)-\mu\right\| \rightarrow 0
\end{aligned}
$$

which implies the result.

$$
\begin{aligned}
\lim _{i \rightarrow \infty} \| \varphi^{\chi}\left(\tau_{i}, \xi_{0}\right) & -\mu\left\|=\lim _{i \rightarrow \infty}\right\| \xi_{i}-\mu \| \\
= & \lim _{i \rightarrow \infty}\left\|\tilde{\xi}_{2 i}-\Upsilon(\mu)\right\| \\
= & \lim _{i \rightarrow \infty}\left\|\psi^{\tilde{\chi}}\left(\tilde{\tau}_{2 i}, \tilde{\xi}_{0}\right)-\Upsilon(\mu)\right\| \rightarrow 0 .
\end{aligned}
$$

$\mathscr{P}=$ EXP: $(\Leftarrow)$ Suppose that

$$
\left\|\psi^{\tilde{\chi}}\left(t, \tilde{\xi}_{0}\right)-\Upsilon(\mu)\right\| \leq M e^{-\alpha t}\left\|\tilde{\xi}_{0}-\Upsilon(\mu)\right\|, \quad t \in \mathcal{T}^{\tilde{\chi}} .
$$

Then for all $t \in\left[\tau_{i}, \tau_{i+1}\right]$,

$$
\begin{aligned}
\| \varphi_{d \chi(i)}\left(t-\tau_{i},\right. & \left.\xi_{i}\right)-\mu \| \\
& =\left\|\psi_{d \tilde{\chi}(2 i)}\left(t-\tau_{i}+i, \tilde{\xi}_{2 i}\right)-\Upsilon(\mu)\right\| \\
& \leq M e^{-\alpha(t+i)}\left\|\tilde{\xi}_{0}-\Upsilon(\mu)\right\| \\
& \leq M e^{-\alpha t}\left\|\xi_{0}-\mu\right\| .
\end{aligned}
$$

$(\Rightarrow)$ Suppose that

$$
\left\|\varphi^{\chi}\left(t, \xi_{0}\right)-\mu\right\| \leq M e^{-\alpha t}\left\|\xi_{0}-\mu\right\|, \quad t \in \mathcal{T}^{\chi}
$$

and define

$$
\begin{aligned}
\beta & =\min _{i \in \Lambda^{\chi}} \beta_{i}, \\
\beta_{i} & =\frac{-\log \frac{\left\|\varphi^{\chi}\left(\tau_{i}, \xi_{0}\right)-\mu\right\|}{M\left\|\xi_{0}-\mu\right\|}}{i-\frac{1}{\alpha} \log \frac{\left\|\varphi^{\chi}\left(\tau_{i}, \xi_{0}\right)-\mu\right\|}{M\left\|\xi_{0}-\mu\right\|}} .
\end{aligned}
$$

It can be verified that $\beta>0, \beta_{i} \leq \alpha$, and for all $t \geq \tilde{\tau}_{2 i}$,

$$
e^{-\alpha(t-i)} \leq e^{-\beta_{i} t} \leq e^{-\beta t} .
$$

From this the result follows since for $t \in\left[\tilde{\tau}_{2 i}, \tilde{\tau}_{2 i+1}\right]$, by (2),

$$
\begin{aligned}
\| \psi^{\tilde{\chi}}\left(t, \tilde{\xi}_{0}\right)-\Upsilon & (\mu) \| \\
& =\left\|\varphi_{d \chi(i)}\left(t-\tau_{i}-i, \xi_{i}\right)-\mu\right\| \\
& \leq M e^{-\alpha(t-i)}\left\|\xi_{0}-\mu\right\| \\
& \leq M e^{-\beta_{i} t}\left\|\xi_{0}-\mu\right\| \\
& \leq M e^{-\beta t}\left\|\tilde{\xi}_{0}-\Upsilon(\mu)\right\|
\end{aligned}
$$

and for all $t \in\left[\tilde{\tau}_{2 i-1}, \tilde{\tau}_{2 i}\right]$,

$$
\begin{aligned}
\| \psi^{\tilde{\chi}}\left(t, \tilde{\xi}_{0}\right)-\Upsilon & (\mu) \| \\
& =\left\|\tilde{\xi}_{2 i}-\Upsilon(\mu)\right\| \\
& \leq M e^{-\beta \tilde{\tau}_{2 i}}\left\|\tilde{\xi}_{0}-\Upsilon(\mu)\right\| \\
& \leq M e^{-\beta t}\left\|\tilde{\xi}_{0}-\Upsilon(\mu)\right\|
\end{aligned}
$$

Therefore,

$$
\left\|\psi^{\tilde{\chi}}\left(t, \tilde{\xi}_{0}\right)-\Upsilon(\mu)\right\| \leq M e^{-\beta t}\left\|\tilde{\xi}_{0}-\Upsilon(\mu)\right\|, \quad t \in \mathcal{T}^{\tilde{\chi}} .
$$

\section{REFERENCES}

[1] A. D. Ames and S. Sastry, "Affine hybrid systems," in Hybrid Systems: Computation and Control, ser. Lecture Notes in Computer Science, R. Alur and G. J. Pappas, Eds., vol. 2993. Springer, 2004, pp. 16-31.

[2] M. S. Branicky, "Stability of hybrid systems: State of the art," in Proceedings of the 36th IEEE Conference on Decision and Control, San Diego, CA, 1997.

[3] R. Hartshorne, Algebraic Geometry, ser. Graduate Texts in Mathematics. Springer, 1977, vol. 52.

[4] M. Heymann, F. Lin, G. Meyer, and S. Resmerita, "Analysis of zeno behaviors in hybrid systems," in Proceedings of the 41st IEEE Conference on Decision and Control, Las Vagas, NV, 2002.

[5] B. Hu, X. Xu, A. Michel, and P. Antsaklis, "Stability analysis for a class of nonlinear switched systems," in Proceedings of the 36th IEEE Conference on Decision and Control, San Diego, CA, 1997.

[6] K. H. Johansson, J. Lygeros, S. Sastry, and M. Egerstedt, "Simulation of zeno hybrid automata," in Proceedings of the 38th IEEE Conference on Decision and Control, Phoenix, AZ, 1999.

[7] D. Liberzon and A. S. Morse, "Basic problems in stablity and design of switched systems," IEEE Control Systems Magazine, vol. 19, no. 5, pp. 59-70, 1999.

[8] M. Rubensson, "Stablity of limit cycles in hybrid systems using discrete-time lyapunov techniques," in Proceedings of the 39th IEEE Conference on Decision and Control, Sydney, Australia, 2000.

[9] S. Sastry, Nonlinear Systems: Analysis, Stability and Control. Springer, 1999.

[10] S. Simic, K. H. Johansson, S. Sastry, and J. Lygeros, "Towards a geometric theory of hybrid systems," in Hybrid Systems: Computation and Control, ser. Lecture Notes in Computer Science, B. Krogh and N. Lynch, Eds., vol. 1790. Springer, 2000, pp. 421-436.

[11] J. Zhang, K. H. Johansson, J. Lygeros, and S. Sastry, "Zeno hybrid systems," Int. J. Robust and Nonlinear Control, vol. 11, no. 2, pp. 435-451, 2001. 